\title{
The boundedness of commutators on locally compact Vilenkin groups
}

\section{Canqin Tang, Qingguo Li and Bolin Ma*}

(Communicated by Hans Triebel)

2000 Mathematics Subject Classification. 43A70, 43A75.

Keywords and phrases. Commutator, Herz-type Triebel-Lizorkin space, Vilenkin group.

Abstract. Let $G$ be a locally compact Vilenkin group. In this paper, the authors investigate the boundedness of commutators of singluar integral operator on Triebel-Lizorkin spaces on $G$. Furthermore, the boundedness on the Herz-type Triebel-Lizorkin spaces are also studied.

\section{Introduction}

The commutators have been studied by many authors for a long time. A well known result which is discovered by Coifman, Rocherg and Weiss ([3], [7], [12]) is that the commutators $[b, T]$ of singular integral operators are bounded on some $L^{p}\left(\mathbb{R}^{n}\right)(1<p<\infty)$ if and only if $b \in B M O$, where $[b, T]$ is defined by $[b, T] f(x)=b(x) T f(x)-T(b f)(x)$. Later, Janson in [6] gave that $[b, T]$ is bounded from $L^{p}\left(\mathbb{R}^{n}\right)$ to $L^{q}\left(\mathbb{R}^{n}\right)$ when $1<p<q<\infty$ if and only if $b \in \operatorname{Lip}_{\beta}$ and $\beta=n\left(\frac{1}{p}-\frac{1}{q}\right)$. In 1995, M. Paluszycński

${ }^{*}$ supported by NSF Grand 10371004 
extended and generalized their results (see [10]). He proved that $[b, T]$ is bounded from $L^{p}\left(\mathbb{R}^{n}\right)$ to $\dot{F}_{p}^{\beta, \infty}\left(\mathbb{R}^{n}\right)$ is equivalent to $b \in \operatorname{Lip}_{\beta}$, where $1<p<\infty, 0<\beta<1$. Motivated by their works, we consider the cases on Vilenkin groups. Moreover, Xu and Yang ([13]) introduced Herz-type Triebel-Lizorkin spaces on $\mathbb{R}^{n}$. In this paper, we continue studying the properties of commutator on Herz-type Triebel-Lizorkin spaces on Vilenkin groups.

In order to state our results more precisely we first introduce some notations and definitions.

Throughout this paper, $G$ will denote a bounded locally compact Vilenkin group, that is, $G$ is a locally compact Abelian group containing a strictly decreasing sequence of compact open subgroups $\left\{G_{n}\right\}_{n=-\infty}^{\infty}$ such that (a) $\cup_{n=-\infty}^{\infty} G_{n}=G$ and $\cap_{n=-\infty}^{\infty} G_{n}=0$; (b) $\sup \left\{\operatorname{order}\left(G_{n} / G_{n+1}\right): n \in \mathbb{Z}\right\}=$ $B<\infty$. Choose Haar measure $d x$ on $G$ so that $\left|G_{0}\right|=1$, where $|A|$ denotes the measure of a measurable subset $A$ of $G$. Let $\left|G_{n}\right|=\left(m_{n}\right)^{-1}$ for each $n \in \mathbb{Z}$. Since $2 m_{n} \leq m_{n+1} \leq B m_{n}$ for each $n \in \mathbb{Z}$, it follows that

$$
\sum_{n=k}^{\infty}\left(m_{n}\right)^{-\alpha} \leq c\left(m_{k}\right)^{-\alpha}
$$

and

$$
\sum_{n=-\infty}^{k}\left(m_{n}\right)^{\alpha} \leq c\left(m_{k}\right)^{\alpha}
$$

for any $\alpha>0, k \in \mathbb{Z}$, where $c$ is a constant independent of $k$. For each $n \in \mathbb{Z}$ we choose elements $z_{l, n} \in G\left(l \in \mathbb{Z}_{+}\right)$so that the subsets $G_{l, n}:=z_{l, n}+G_{n}$ of $G$ satisfy $G_{k, n} \cap G_{l, n}=\phi$ if $k \neq l$ and $\cup_{l=0}^{\infty} G_{l, n}=G$; moreover, we choose $z_{0, n}$ such that $G_{0, n}=G_{n}$. We now define the function $d: G \times G \rightarrow R$ by $d(x, y)=0$ if $x-y=0$ and $d(x, y)=\left(m_{n}\right)^{-1}$ if $x-y \in G_{n} \backslash G_{n+1}$. Then $d$ is a metric on $G$ and the topology on $G$ generated by this metric is the same as the original topology on $G$. For $x \in G$, set $|x|=d(x, 0)$. Then $|x|=\left(m_{n}\right)^{-1}$ if and only if $x \in G_{n} \backslash G_{n+1}$. Let $S(G)$ be the space of test functions and $S^{\prime}(G)$ be the distribution space on $G$. Set $\varphi_{n}=m_{n} \chi_{G_{n}}-m_{n+1} \chi_{G_{n+1}}$, where $\chi_{G_{n}}$ is the characteristic function of $G_{n}$.

Definition 1.1. Let $0 \leq \alpha<\infty, 0<p, q<\infty$, the homogeneous Herz spaces $\dot{K}_{q}^{\alpha, p}(G)$ are defined by $\dot{K}_{q}^{\alpha, p}(G)=\left\{f: f\right.$ is a measurable function on $G$ with $\left.\|f\|_{\dot{K}_{q}^{\alpha, p}(G)}<\infty\right\}$, where

$$
\|f\|_{\dot{K}_{q}^{\alpha, p}(G)}=\left\{\sum_{j=-\infty}^{\infty} m_{l}^{-\alpha p}\left\|f \chi_{G_{l} \backslash G_{l+1}}\right\|_{L^{q}(G)}^{p}\right\}^{1 / p} .
$$

Before defining the Herz-type Triebel-Lizorkin spaces on $G$, we give the notes of a second space of test functions and distributions. We refer to [4] 
for details. Let

$$
Z(G)=\left\{\psi \in S(G): \hat{\psi}(0)=\int_{G} \psi(t) d t=0\right\}
$$

and define the convergence in $Z(G)$ to be like in $S(G)$. Let $Z^{\prime}(G)$ be the space of linear functionals on $Z(G)$ with convergence in $Z^{\prime}(G)$ defined as in $S^{\prime}(G)$ and $\varrho$ denotes the set of constant distributions in $S^{\prime}(G)$.

Definition 1.2. Let $\alpha \in \mathbb{R}, 0<p, q \leq \infty$. Then

$\dot{F}_{q}^{\alpha, p}(G)=\left\{f \in Z^{\prime}(G):\|f\|_{\dot{F}_{q}^{\alpha, p}(G)}:=\left\|\left(\sum_{n=-\infty}^{\infty}\left(m_{n}\right)^{\alpha q}\left|f * \varphi_{n}\right|^{q}\right)^{1 / q}\right\|_{p}<\infty\right\}$,

with the usual modification if $q=\infty$.

Definition 1.3. Let $\alpha \in \mathbb{R}, 0<p, q \leq \infty$. Then

$$
\begin{aligned}
& \dot{K}_{q}^{\alpha, p} \dot{F}_{\beta}^{s}(G)=\left\{f \in Z^{\prime}(G):\|f\|_{\dot{K}_{q}^{\alpha, p} \dot{F}_{\beta}^{s}(G)}\right. \\
&:=\|\left(\sum_{n=-\infty}^{\infty}\left(\left(m_{n}\right)^{s \beta}\left|f * \varphi_{n}\right|^{\beta}\right)^{1 / \beta} \|_{\dot{K}_{q}^{\alpha, p}}<\infty\right\},
\end{aligned}
$$

with the usual modification if $\beta=\infty$.

Definition 1.4. Let $0 \leq \alpha<\infty$ and $0<q<\infty$. A function $b \in L^{q}(G)$ is said to be a central $(\alpha, q)$-block if it satisfies

(i) for some $n \in \mathbb{Z}, \operatorname{supp} b \subset G_{n}$;

(ii) $\|b\|_{L^{q}(G)} \leq m_{n}^{\alpha}$;

Here $\operatorname{supp} b$ denote the support of function $b(x)$.

By the conclusion in [9], the functions $f$ in $\dot{K}_{q}^{\alpha, p}(G)$ have the following characterization.

Proposition $1.1([9])$. Let $0<\alpha<\infty, 1 \leq q<\infty, 0<p<\infty$. Then $f \in \dot{K}_{q}^{\alpha, p}(G)$ if and only if $f=\sum_{j=-\infty}^{\infty} \lambda_{j} b_{j}$ in distributional sense, where each $b_{j}$ is a central $(\alpha, q)$-block and $\sum_{j=-\infty}^{\infty}\left|\lambda_{j}\right|^{p}<\infty$. Moreover,

$$
\|f\|_{\dot{K}_{q}^{\alpha, p}(G)} \sim \inf \left\{\left(\sum_{j=-\infty}^{\infty}\left|\lambda_{j}\right|^{p}\right)^{\frac{1}{p}}\right\}
$$

with the infimum taken over all decompositions of $f$ as above. 
212 Boundedness of commutators on locally compact Vilenkin groups

In order to study the boundedness of commutators, we also need to define Lipschitz spaces. For $x_{0} \in G$, set $I_{j}=x_{0}+G_{j}$,

Definition 1.5 ([8,10,2]). For $0<\beta<1,1 \leq q \leq \infty$, the Lipschtiz spaces $\operatorname{Lip}_{\beta}(G)$ are defined by

$$
\operatorname{Lip}_{\beta}(G)=\left\{f \mid\|f\|_{\beta}<\infty\right\}
$$

where

$$
\begin{aligned}
\|f\|_{\beta} & =\sup _{I_{j} \ni x} \frac{1}{\left|I_{j}\right|^{1+\beta}} \int_{I_{j}}\left|f(y)-f_{I_{j}}\right| d y \\
& =\sup _{I_{j} \ni x} \frac{1}{\left|I_{j}\right|^{\beta}}\left(\frac{1}{\left|I_{j}\right|} \int_{I_{j}}\left|f(y)-f_{I_{j}}\right|^{q} d y\right)^{1 / q},
\end{aligned}
$$

with the $f_{I_{j}}$ being the average of $f$ over $I_{j}$ and the usual modification in the case of $q=\infty$.

Our main results are the following.

Theorem 1.1. Let $1<p<\infty, 0<\beta<1, b \in \operatorname{Lip}_{\beta}(G)$. If $T$ is a singular integral operator, then $[b, T]$ is a bounded operator from $L^{p}(G)$ to $\dot{F}_{p}^{\beta, \infty}(G)$.

Theorem 1.2. Let $1<q<\infty, 0<\beta<1, b \in \operatorname{Lip}_{\beta}, 0<\alpha<1-\frac{1}{q}$. If $T$ is a singular integral operator, then $[b, T]$ is a bounded operator from $\dot{K}_{q}^{\alpha, p}$ to $\dot{K}_{q}^{\alpha, p} \dot{F}_{\beta}^{\infty}$.

As we will show that

$\frac{1}{\left|I_{j}\right|^{1+\beta}} \int_{I_{j}}\left|[b, T] f-([b, T] f)_{I_{j}}\right| \leq C\|b\|_{\beta}\left[M(T f)(x)+M_{r}(f)(x)+M(f)(x)\right]$,

taking the supremum over all $I_{j}$ such that $x \in I_{j}$, and then consider the $\dot{K}_{q}^{\alpha, p}$ norm of both sides, it is obviously to see that Theorem 1.2 is a consequence of the following theorem.

Theorem 1.3. Let $1 \leq r<q<\infty$. If a sublinear operator $T$ satisfies

$$
|T f(x)| \leq C\left(\int_{\operatorname{supp} f} \frac{|f(y)|^{r}}{|x-y|} d y\right)^{1 / r} \quad \text { for } \quad x \notin \operatorname{supp} f
$$

for all $f \in L^{q}(G)$ with compact support and $T$ maps $L^{q}(G)$ into $L^{q}(G)$, then $T$ is bounded on $\dot{K}_{q}^{\alpha, p}(G)$, provided $0<\alpha<\frac{1}{r}-\frac{1}{q}$ and $0<p \leq \infty$.

In section 2, we'll give the proof of Theorem 1.1. And Theorem 1.3 will be proved in section 3 . 


\section{Boundedness of the commutator on the Triebel-Lizorkin spaces}

Let $T$ be a singular integral operator, i. e.

$$
T f(x)=\int_{G} K(x-y) f(y) d y
$$

where $K(x-y)$ is the kernel and $\left|K(x-y)-K\left(x_{0}-y\right)\right| \leq c \frac{\left|x-x_{0}\right|}{\left|y-x_{0}\right|^{2}}$. Before we start to show our theorems, we first give some equivalent norms of Triebel-Lizorkin spaces. In [1], A. Seeger gave the characterization of Triebel-Lizorkin spaces $\dot{F}_{\alpha}^{p, q}$ on $\mathbb{R}^{n}$ via means of maximal functions, which is introduced by Devore and Sharpley in [5] and Christ in [2]. Paralleled to the situation in [1], using the maximal operator, we can obtain the following lemma.

Write

$$
D^{\beta} f(x)=\sup _{j} m_{j}^{\beta} \frac{1}{\left|\left\{y \mid y-x \in I_{j}\right\}\right|} \int_{\left\{y \mid y-x \in I_{j}\right\}}\left|f(y)-f_{I_{j}}\right| d y,
$$

and

$$
S^{\beta} f(x)=\sup _{x \in I_{j}} m_{j}^{\beta} \frac{1}{\left|I_{j}\right|} \int_{I_{j}}|f(x+h)-f(x)| d h .
$$

Lemma 2.1. For $0<\beta<1,1<p<\infty$, we have

$$
\left\|D^{\beta} f\right\|_{p} \sim\|f\|_{\dot{F}_{p}^{\beta, \infty}(G)} \sim\left\|S^{\beta} f\right\|_{p} .
$$

Proof. According to the definition, the average over $I_{j}$ is dominated as

$$
\begin{aligned}
\frac{1}{\left|I_{j}\right|} \int_{I_{j}}|f(x+h)-f(x)| d h \leq & \frac{1}{\left|I_{j}\right|} \int_{I_{j}}\left|f(x+h)-f_{I_{j}}\right| d h \\
& +\frac{1}{\left|I_{j}\right|} \int_{I_{j}}\left|f(x)-f_{I_{j}}\right| d h \\
= & A+\left|f(x)-f_{I_{j}}\right| .
\end{aligned}
$$

Replacing $x+h$ by $y, A$ is easily controlled by

$$
\frac{1}{\left|\left\{y \mid y-x \in I_{j}\right\}\right|} \int_{\left\{y \mid y-x \in I_{j}\right\}}\left|f(y)-f_{I_{j}}\right| d y .
$$

To deal with the second term, using the differential theorem of integral, we have

$$
\left|f(x)-f_{I_{j}}\right|=\left|\lim _{k \rightarrow-\infty, x \in I_{k}} \frac{1}{\left|I_{k}\right|} \int_{I_{k}} f(y) d y-f_{I_{j}}\right|
$$


214 Boundedness of commutators on locally compact Vilenkin groups

$$
\begin{aligned}
& \leq \sum_{k=-\infty}^{j}\left|f_{I_{k+1}}-f_{I_{k}}\right| \\
& \leq B \sum_{k=-\infty}^{j} \frac{1}{\left|I_{k}\right|} \int_{I_{k}}\left|f(y)-f_{I_{k}}\right| d y .
\end{aligned}
$$

Since $0<\beta<1, k \leq j$ and the domination of (2.1), we have

$$
\begin{aligned}
& \sup _{x \in I_{j}} m_{j}^{\beta} \frac{1}{\left|I_{j}\right|} \int_{I_{j}}|f(x+h)-f(x)| d h \\
& \leq C \sup _{x \in I_{j}} m_{j}^{\beta} \sum_{k=-\infty}^{j} \frac{1}{\left|\left\{y \mid y-x \in I_{k}\right\}\right|} \int_{\left\{y \mid y-x \in I_{k}\right\}}\left|f(y)-f_{I_{k}}\right| d y \\
& \leq C \sup _{x \in I_{j}} \frac{1}{\left|\left\{y \mid y-x \in I_{j}\right\}\right|} \int_{\left\{y \mid y-x \in I_{j}\right\}}\left|f(y)-f_{I_{j}}\right| d y \sum_{k=-\infty}^{j} m_{j}^{\beta}\left(\frac{m_{k}}{m_{j}}\right) \\
& \leq C \sup _{j} m_{j}^{\beta} \frac{1}{\left|\left\{y \mid y-x \in I_{j}\right\}\right|} \int_{\left\{y \mid y-x \in I_{j}\right\}}\left|f(y)-f_{I_{j}}\right| d y .
\end{aligned}
$$

Hence $S^{\beta} f \leq C D^{\beta} f$.

$$
\begin{aligned}
& \text { Next, we have } f=\sum_{k=-\infty}^{j} \varphi_{k} * f+\sum_{k=j+1}^{\infty} \varphi_{k} * f=f_{1}+f_{2} \text {. It is easy to see } \\
& \frac{1}{\left|\left\{y \mid y-x \in I_{j}\right\}\right|} \int_{\left\{y \mid y-x \in I_{j}\right\}}\left|f_{2}(y)-f_{2_{I_{j}}}\right| d y \leq M f_{2},
\end{aligned}
$$

where $M$ is maximal operator. Consequently,

$$
\begin{aligned}
& \left\|\sup _{j} m_{j}^{\beta} \frac{1}{\left|\left\{y \mid y-x \in I_{j}\right\}\right|} \int_{\left\{y \mid y-x \in I_{j}\right\}}\left|f_{2}(y)-f_{2_{I_{j}}}\right| d y\right\|_{p} \\
& \leq\left\|\sup _{j} m_{j}^{\beta} M f_{2}\right\|_{p} \\
& \leq C\left\|\sup _{j} m_{j}^{\beta} \sum_{k=j+1}^{\infty} \varphi_{k} * f\right\|_{p} \\
& \leq C\left\|\sup _{k} m_{k}^{\beta}\left|\varphi_{k} * f\right| \sum_{k=j+1}^{\infty} m_{j}^{\beta} m_{k}^{-\beta}\right\|_{p} \\
& \leq C\left\|\sup _{k} m_{k}^{\beta}\left|\varphi_{k} * f\right|\right\|_{p} .
\end{aligned}
$$


As $f_{1}=\sum_{k=-\infty}^{j} \varphi_{k} * f$

$$
\begin{aligned}
& \left\|\sup m_{j}^{\beta} \frac{1}{\left|\left\{y \mid y-x \in I_{j}\right\}\right|} \int_{\left\{y \mid y-x \in I_{j}\right\}}\left|f_{1}(y)-f_{1_{I_{j}}}\right| d y\right\|_{p} \\
& \leq C\left\|\sup _{j} m_{j}^{\beta} \frac{1}{\left|\left\{y \mid y-x \in I_{j}\right\}\right|} \int_{\left\{y \mid y-x \in I_{j}\right\}}\left|\sum_{k=-\infty}^{j} \frac{m_{k}}{m_{j}} \sigma_{k}^{*} f(y)\right| d y\right\|_{p} \\
& \leq C\left\|\sup _{k} m_{k} \sigma_{k}^{*} f\right\|_{p} \\
& \leq C\left\|\sup _{k} m_{k}\left|\varphi_{k} * f\right|\right\|_{p}
\end{aligned}
$$

where $\sigma_{k}^{*} f(y)=\sup _{z \in G} \frac{\left|\varphi_{k} * f(y-z)\right|}{m_{k}|z|}$. Thus, $\left\|D^{\beta} f\right\|_{p} \leq C\|f\|_{\dot{F}_{p}^{\beta, \infty}}$.

Since $\varphi_{k}=\triangle_{k}-\triangle_{k+1}, \triangle_{k}=m_{k} \chi_{G_{k}}$, where $\chi$ is the characteristic function of a subset of $G$, we can easily show that $\|f\|_{\dot{F}_{p}^{\beta, \infty}} \leq C\left\|S^{\beta} f\right\|_{p}$. Lemma 2.1 is proved.

From Lemma 2.1, we know that

$$
\|f\|_{\dot{F}_{p}^{\beta, \infty}} \simeq\left\|\sup _{I \ni x} \frac{1}{|I|^{1+\beta}} \int_{I}\left|f-f_{I}\right|\right\|_{p} .
$$

Now we start to prove Theorem 1.1.

Proof of Theorem 1.1. Let $I_{j}=x_{0}+G_{j}$ and $x \in I_{j}$. For a fixed $f \in L^{p}$, write $f_{1}=f \chi_{I_{j}}$ and $f_{2}=f-f_{1}$. It is easy to see that $[b, T] f=\left[b-b_{I_{j}}, T\right] f$, and so we have

$$
\begin{aligned}
& \frac{1}{\left|I_{j}\right|^{1+\beta}} \int_{I_{j}}\left|[b, T] f(x)-([b, T] f)_{I_{j}}\right| d x \\
\leq & \frac{1}{\left|I_{j}\right|^{1+\beta}} \int_{I_{j}}\left|\left[b-b_{I_{j}}, T\right] f(x)-\left(\left[b-b_{I_{j}}, T\right] f\right)_{I_{j}}\right| d x \\
\leq & C \frac{1}{\left|I_{j}\right|^{1+\beta}} \int_{I_{j}}\left|\left[b-b_{I_{j}}, T\right] f(x)-T\left(\left(b-b_{I_{j}}\right) f_{2}\right)\left(x_{0}\right)\right| d x \\
\leq & C \frac{1}{\left|I_{j}\right|^{1+\beta}} \int_{I_{j}}\left|\left(b-b_{I_{j}}\right) T f(x)\right|+C \frac{1}{\left|I_{j}\right|^{1+\beta}} \int_{I_{j}}\left|T\left(\left(b-b_{I_{j}}\right) f_{1}(x)\right)\right| \\
& +C \frac{1}{\left|I_{j}\right|^{\beta}} \sup _{y \in I_{j}}\left|T\left(\left(b-b_{I_{j}}\right) f_{2}\right)(y)-T\left(\left(b-b_{I_{j}}\right) f_{2}\right)\left(x_{0}\right)\right| \\
= & L_{1}+L_{2}+L_{3} .
\end{aligned}
$$

We give the estimations for $L_{1}, L_{2}$ and $L_{3}$ respectively. By the definition of the Lipschitz space, the $L_{1}$ is easily to be dominated by the maximal 
216 Boundedness of commutators on locally compact Vilenkin groups operator, that is

$$
\begin{aligned}
\frac{1}{\left|I_{j}\right|^{1+\beta}} \int_{I_{j}}\left|\left(b-b_{I_{j}}\right) T f\right| & \leq \frac{1}{\left|I_{j}\right|^{\beta}} \sup _{y \in I_{j}}\left|b(y)-b_{I_{j}}\right| \frac{1}{\left|I_{j}\right|} \int_{I_{j}}|T f(y)| d y \\
& \leq C\|b\|_{\beta} M(T f)(x) .
\end{aligned}
$$

To estimate $L_{2}$, choosing $r>1$, we have

$\frac{1}{\left|I_{j}\right|}\left\|\left(b-b_{I_{j}}\right) f_{1}\right\|_{r}^{r} \leq C \sup _{y \in I_{j}}\left|b(y)-b_{I_{j}}\right|^{r} M_{r}^{r}(f)(x) \leq C m_{j}^{-\beta r}\|b\|_{\beta}^{r} M_{r}^{r}(f)(x)$.

Consequently,

$$
\left\|\left(b-b_{I_{j}}\right) f_{1}\right\|_{r} \leq C m_{j}^{-\beta-\frac{1}{r}}\|b\|_{\beta} M_{r}(f)(x) .
$$

Then, using Hölder inequality and $L^{r}$-boundedness of singular operator, we get

$$
\begin{aligned}
L_{2} & =C \frac{1}{\left|I_{j}\right|^{1+\beta}} \int_{I_{j}}\left|T\left(\left(b-b_{I_{j}}\right) f_{1}\right)\right| \\
& \leq C \frac{1}{\left|I_{j}\right|^{1+\beta}}\left\|T\left(\left(b-b_{I_{j}}\right) f_{1}\right)\right\|_{r}\left|I_{j}\right|^{1-\frac{1}{r}} \\
& \leq C \frac{1}{\left|I_{j}\right|^{1+\beta}}\left\|\left(b-b_{I_{j}}\right) f_{1}\right\|_{r}\left|I_{j}\right|^{1-\frac{1}{r}} \\
& \leq C\|b\|_{\beta} M_{r} f(x) .
\end{aligned}
$$

Now we turn to deal with $L_{3}$. By the hypothesis on the kernel $K$, we have

$$
\begin{aligned}
& \left|T\left(\left(b-b_{I_{j}}\right) f_{2}\right)(y)-T\left(\left(b-b_{I_{j}}\right) f_{2}\right)\left(x_{0}\right)\right| \\
& \quad=\left|\int_{G}\left(K(y-z)-K\left(x_{0}-z\right)\right)\left(b(z)-b_{I_{j}}\right) f_{2}(z) d z\right| \\
& \leq C \int_{\left\{z \mid z-x_{0} \in G \backslash G_{j}\right\}} \frac{\left|y-x_{0}\right|}{\left|x_{0}-z\right|^{2}}\left|b(z)-b_{I_{j}}\right||f(z)| d z \\
& \quad \leq C m_{j}^{-1} \sum_{k=-\infty}^{j-1} m_{k}^{2} \int_{\left\{z \mid z-x_{0} \in G_{k} \backslash G_{k+1}\right\}}\left(\left|b(z)-b_{I_{k}}\right|+\left|b_{I_{k}}-b_{I_{j}}\right|\right)|f(z)| d z .
\end{aligned}
$$

It is easy to see

$$
\begin{aligned}
& \int_{\left\{z \mid z-x_{0} \in G_{k} \backslash G_{k+1}\right\}}\left|b(z)-b_{I_{k}}\right||f(z)| d z \\
& \quad \leq \sup _{z \in\left\{z \mid z-x_{0} \in G_{k} \backslash G_{k+1}\right\}}\left|b(z)-b_{I_{k}}\right| \int_{\left\{z \mid z-x_{0} \in G_{k} \backslash G_{k+1}\right\}}|f(z)| d z \\
& \leq\|b\|_{\beta} m_{k}^{-\beta-1}(M f)(x)
\end{aligned}
$$


and

$$
\begin{aligned}
\int_{\left\{z \mid z-x_{0} \in G_{k} \backslash G_{k+1}\right\}}\left|b_{I_{k}}-b_{I_{j}}\right||f(z)| d z & \leq\left|b_{I_{k}}-b_{I_{j}}\right| m_{k}^{-1}(M f)(x) \\
& \leq\|b\|_{\beta} m_{k}^{-\beta-1}(M f)(x),
\end{aligned}
$$

where $\left|b_{I_{k}}-b_{I_{j}}\right|$ 's have the following estimates

$$
\begin{aligned}
\left|b_{I_{k}}-b_{I_{j}}\right| & =\left|\frac{1}{\left|I_{j}\right|} \int_{I_{j}} b(z) d z-b_{I_{k}}\right| \\
& \leq \frac{1}{\left|I_{j}\right|} \int_{I_{j}}\left|b(z)-b_{I_{k}}\right| d z \\
& \leq \sup _{z \in I_{j}}\left|b(z)-b_{I_{k}}\right| \\
& \leq \sup _{z \in I_{k}}\left|b(z)-b_{I_{k}}\right| \\
& \leq\|b\|_{\beta} m_{k}^{-\beta}
\end{aligned}
$$

for $k \leq j-1$ and $I_{k} \supset I_{j}$. Thus, we obtain

$$
\begin{aligned}
& \left|T\left(\left(b-b_{I_{j}}\right) f_{2}\right)(y)-T\left(\left(b-b_{I_{j}}\right) f_{2}\right)\left(x_{0}\right)\right| \\
& \quad \leq C\|b\|_{\beta} m_{j}^{-1} \sum_{k=-\infty}^{j-1} m_{k}^{2} m_{k}^{-\beta-1} M f(x) \\
& \quad \leq C\|b\|_{\beta} m_{j}^{-\beta} M f(x)
\end{aligned}
$$

and we can deduce that $L_{3} \leq C\|b\|_{\beta} M f(x)$.

Taking account of all the estimates for $L_{1}, L_{2}$ and $L_{3}$ together, we obtain

$\frac{1}{\left|I_{j}\right|^{1+\beta}} \int_{I_{j}}\left|[b, T] f-([b, T] f)_{I_{j}}\right| \leq C\|b\|_{\beta}\left(M(T f)(x)+M_{r}(f)(x)+M(f)(x)\right)$.

Taking the supremum over all $I_{j}$ such that $x \in I_{j}$, and the $L_{p}$-norm on both sides, and using Lemma 2.1 we conclude that

$$
\|[b, T] f\|_{\dot{F}_{p}^{\beta, \infty}} \leq C\|b\|_{\beta}\|f\|_{p} .
$$

Thus, Theorem 1.1 is proved.

\section{Boundedness of some sublinear operators on Herz space}

In this section, we will consider the boundedness of some sublinear operators on Herz space.

Proof of Theorem 1.3. We first prove the case when $r=1$. Let $f \in \dot{K}_{q}^{\alpha, p}(G)$. Then by Proposition 1.1, we have $f(x)=\sum_{j=-\infty}^{\infty} \lambda_{j} b_{j}(x)$, 
218 Boundedness of commutators on locally compact Vilenkin groups

where $\left(\sum_{j=-\infty}^{\infty}\left|\lambda_{j}\right|^{p}\right)^{\frac{1}{p}} \leq C\|f\|_{\dot{K}_{q}^{\alpha, p}}$, and $b_{j}$ 's are $(\alpha, q)$-block supported in $G_{j}, j \in \mathbb{Z}$. Let $\chi_{n}=\chi_{G_{n} \backslash G_{n+1}}$. Since $T$ is a sublinear operator, we have

$$
\begin{aligned}
\|T f\|_{\dot{K}_{q}^{\alpha, p}}= & \left\{\sum_{n=-\infty}^{\infty} m_{n}^{-\alpha p}\left\|(T f) \chi_{n}\right\|_{q}^{p}\right\}^{1 / p} \\
\leq & \left\{\sum_{n=-\infty}^{\infty} m_{n}^{-\alpha p}\left(\sum_{j=-\infty}^{n}\left|\lambda_{j}\right|\left\|\left(T b_{j}\right) \chi_{n}\right\|_{q}\right)^{p}\right. \\
& \left.+\sum_{n=-\infty}^{\infty} m_{n}^{-\alpha p}\left(\sum_{j=n+1}^{\infty}\left|\lambda_{j}\right|\left\|\left(T b_{j}\right) \chi_{n}\right\|_{q}\right)^{p}\right\}^{1 / p} \\
= & \left(L_{1}+L_{2}\right)^{1 / p} .
\end{aligned}
$$

In the case of $0<p \leq 1$, using $L^{q}$-boundedness of $T$, we get

$$
\begin{aligned}
L_{1} & =\sum_{n=-\infty}^{\infty} m_{n}^{-\alpha p}\left(\sum_{j=-\infty}^{n}\left|\lambda_{j}\right|\left\|\left(T b_{j}\right) \chi_{n}\right\|_{q}\right)^{p} \\
& \leq C \sum_{n=-\infty}^{\infty} m_{n}^{-\alpha p}\left(\sum_{j=-\infty}^{n}\left|\lambda_{j}\right|^{p}\left\|b_{j}\right\|_{q}^{p}\right) \\
& \leq C \sum_{j=-\infty}^{\infty}\left|\lambda_{j}\right|^{p} m_{j}^{\alpha p}\left(\sum_{n=j}^{\infty} m_{n}^{-\alpha p}\right) \\
& \leq C \sum_{j=-\infty}^{\infty}\left|\lambda_{j}\right|^{p}<\infty .
\end{aligned}
$$

In the case of $1<p \leq \infty$, by Hölder's inequality and the $L^{q}$ boundedness of $T$, we obtain

$$
\begin{aligned}
L_{1} & \leq C \sum_{n=-\infty}^{\infty} m_{n}^{-\alpha p}\left(\sum_{j=-\infty}^{n}\left|\lambda_{j}\right|^{p}\left\|b_{j}\right\|_{q}^{p}\right) \\
& \leq C \sum_{n=-\infty}^{\infty}\left\{\left(\sum_{j=-\infty}^{n}\left|\lambda_{j}\right|^{p}\left(\frac{m_{j}}{m_{n}}\right)^{\alpha p / 2}\right)\left(\sum_{j=-\infty}^{n}\left(\frac{m_{j}}{m_{n}}\right)^{\alpha p^{\prime} / 2}\right)^{\frac{p}{p^{\prime}}}\right\} \\
& \leq C \sum_{j=-\infty}^{\infty}\left|\lambda_{j}\right|^{p}<\infty
\end{aligned}
$$

where $p^{\prime}$ is the conjugate index of $p$.

Now we turn to estimate $L_{2}$. For $j \geq n+1, G_{j} \subset G_{n+1} \subset G_{n}$. If $x \in G_{n} \backslash G_{n+1}$ and $y \in G_{j}$, then $x-y \in G_{n} \backslash G_{n+1}$. By the condition of $T$, 
we have

$$
\begin{aligned}
\left\|\left(T b_{j}\right) \chi_{n}\right\|_{q} & =\left(\int_{G_{n} \backslash G_{n+1}}\left|T b_{j}\right|^{q} d x\right)^{1 / q} \\
& \leq C\left(\int_{G_{n} \backslash G_{n+1}}\left(\int_{G_{j}} \frac{\left|b_{j}(y)\right|}{|x-y|} d y\right)^{q} d x\right)^{1 / q} \\
& \leq C m_{n}^{1-\frac{1}{q}}\left\|b_{j}\right\|_{q}\left|G_{j}\right|^{\frac{1}{q^{\prime}}} \\
& \leq C m_{n}^{1-\frac{1}{q}} m_{j}^{\alpha} m_{j}^{-\left(1-\frac{1}{q}\right)}
\end{aligned}
$$

Similar to the estimation of $L_{1}$, we also consider two cases.

Case A: $0<p \leq 1$. In this case,

$$
\begin{aligned}
L_{2} & =\sum_{n=-\infty}^{\infty} m_{n}^{-\alpha p}\left(\sum_{j=n+1}^{\infty}\left|\lambda_{j}\right|\left\|\left(T b_{j}\right) \chi_{n}\right\|_{q}\right)^{p} \\
& \leq C \sum_{n=-\infty}^{\infty} m_{n}^{-\alpha p}\left(\sum_{j=n+1}^{\infty}\left|\lambda_{j}\right|^{p}\left(\frac{m_{n}}{m_{j}}\right)^{\left(1-\frac{1}{q}\right) p} m_{j}^{\alpha p}\right) \\
& \leq C \sum_{j=-\infty}^{\infty}\left|\lambda_{j}\right|^{p} \sum_{n=-\infty}^{j-1}\left(\frac{m_{n}}{m_{j}}\right)^{\left(1-\frac{1}{q}-\alpha\right) p} \\
& \leq C \sum_{j=-\infty}^{\infty}\left|\lambda_{j}\right|^{p}<\infty
\end{aligned}
$$

since $0<\alpha<1-1 / q$.

Case B: $1<p \leq \infty$. Let $\frac{1}{p}+\frac{1}{p^{\prime}}=1$. Using the Hölder's inequality,

$$
\begin{aligned}
L_{2} \leq C \sum_{n=-\infty}^{\infty} m_{n}^{-\alpha p}\left(\sum_{j=n+1}^{\infty}\left|\lambda_{j}\right|\left(\frac{m_{n}}{m_{j}}\right)^{1-\frac{1}{q}} m_{j}^{\alpha}\right)^{p} \\
\leq C \sum_{n=-\infty}^{\infty}\left(\sum_{j=n+1}^{\infty}\left|\lambda_{j}\right|\left(\frac{m_{n}}{m_{j}}\right)^{\left(1-\frac{1}{q}-\alpha\right)}\right)^{p} \\
\leq C \sum_{n=-\infty}^{\infty}\left(\sum_{j=n+1}^{\infty}\left|\lambda_{j}\right|^{p}\left(\frac{m_{n}}{m_{j}}\right)^{\left(1-\frac{1}{q}-\alpha\right) p / 2}\right) \\
\left.\quad \times\left(\sum_{j=n+1}^{\infty}\left(\frac{m_{n}}{m_{j}}\right)^{\left(1-\frac{1}{q}-\alpha\right) p^{\prime} / 2}\right)^{\frac{p}{p^{\prime}}}\right)\left.^{\infty}\left|\sum_{j=-\infty}^{\infty}\right| \lambda_{j}\right|^{p}<\infty .
\end{aligned}
$$


220 Boundedness of commutators on locally compact Vilenkin groups

Using all the estimations above, we obtain

$$
\|T f\|_{\dot{K}_{q}^{\alpha, p}} \leq C\left(\sum_{j=-\infty}^{\infty}\left|\lambda_{j}\right|^{p}\right)^{1 / p} \leq C\|f\|_{\dot{K}_{q}^{\alpha, p}} .
$$

When $r>1$, similar to the proof of $r=1$, we can decompose $\|T f\|_{\dot{K}_{q}^{\alpha, p}}$ into two parts which are dominated by $\left(L_{1}+L_{2}\right)^{1 / p}$. The estimate for $L_{1}$ is easily obtained as in the proof of $r=1$. We only consider to estimate $L_{2}$ now. By the hypothesis (1.1), we have

$$
\begin{aligned}
\left\|\left(T b_{j}\right) \chi_{n}\right\|_{q} & \leq C\left(\int_{G_{n} \backslash G_{n+1}}\left(\int_{G_{j}} \frac{\left|b_{j}(y)\right|^{r}}{|x-y|} d y\right)^{\frac{q}{r}} d x\right)^{1 / q} \\
& \leq C m_{n}^{\frac{1}{r}-\frac{1}{q}}\left(\int_{G_{j}}\left|b_{j}(y)\right|^{r} d y\right)^{1 / r} \\
& \leq C m_{n}^{\frac{1}{r}-\frac{1}{q}} m_{j}^{\alpha+\frac{1}{q}-\frac{1}{r}} .
\end{aligned}
$$

So, if $0<p \leq 1$, then

$$
\begin{aligned}
L_{2} & \leq C \sum_{n=-\infty}^{\infty} m_{n}^{-\alpha p}\left(\sum_{j=n+1}^{\infty}\left|\lambda_{j}\right|^{p}\left(\frac{m_{n}}{m_{j}}\right)^{\left(\frac{1}{r}-\frac{1}{q}\right) p} m_{j}^{\alpha p}\right) \\
& \leq C \sum_{j=-\infty}^{\infty}\left|\lambda_{j}\right|^{p}\left(\sum_{n=-\infty}^{j-1}\left(\frac{m_{n}}{m_{j}}\right)^{\left(\frac{1}{r}-\frac{1}{q}-\alpha\right) p}\right) \\
& \leq C \sum_{j=-\infty}^{\infty}\left|\lambda_{j}\right|^{p}<\infty
\end{aligned}
$$

where $0<\alpha<\frac{1}{r}-\frac{1}{q}$.

If $1<p \leq \infty$, then

$$
\begin{aligned}
L_{2} \leq & C \sum_{n=-\infty}^{\infty} m_{n}^{-\alpha p}\left(\sum_{j=n+1}^{\infty}\left|\lambda_{j}\right|\left(\frac{m_{n}}{m_{j}}\right)^{\left(\frac{1}{r}-\frac{1}{q}\right)} m_{j}^{\alpha}\right)^{p} \\
\leq & C \sum_{n=-\infty}^{\infty}\left(\sum_{j=n+1}^{\infty}\left|\lambda_{j}\right|\left(\frac{m_{n}}{m_{j}}\right)^{\frac{1}{r}-\frac{1}{q}-\alpha}\right)^{p} \\
\leq & C \sum_{n=-\infty}^{\infty}\left(\sum_{j=n+1}^{\infty}\left|\lambda_{j}\right|^{p}\left(\frac{m_{n}}{m_{j}}\right)^{\left(\frac{1}{r}-\frac{1}{q}-\alpha\right) p / 2}\right) \\
& \times\left(\sum_{j=n+1}^{\infty}\left(\frac{m_{n}}{m_{j}}\right)^{\left(\frac{1}{r}-\frac{1}{q}-\alpha\right) p^{\prime} / 2}\right)^{\frac{p}{p^{\prime}}}
\end{aligned}
$$




$$
\leq C \sum_{j=-\infty}^{\infty}\left|\lambda_{j}\right|^{p}<\infty
$$

Combining the estimations of $L_{1}$ with $L_{2}$, we can deduce that

$$
\|T f\|_{\dot{K}_{q}^{\alpha, p}} \leq C\left(\sum_{j=-\infty}^{\infty}\left|\lambda_{j}\right|^{p}\right)^{1 / p} \leq C\|f\|_{\dot{K}_{q}^{\alpha, p} .}
$$

This finishes the proof of Theorem 1.3.

\section{References}

[1] A. Seeger, A note on Triebel-Lizorkin spaces, Approximation and Function Spaces, PWN, Warsaw, 1989, 391-400.

[2] M. Christ, The extension problem for certain function spaces involving fractional orders of differentiability, Ark. Mat, 22 (1984), 63-81.

[3] R. R. Coifman, R. Rochberg and G. Weiss, Factorization theorems for Hardy spaces in several variables, Ann. of Math., 103 (1976), 611-635.

[4] C. W. Onnewer and W. Y. Su, Homogeneous Besov spaces on locally compact Vilenkin groups, Studia Math., T. XCIII. (1989), 17-39.

[5] R. A. Devore and R. C. Sharpley, Maximal functions measuring smoothness, Mem. Amer. Math. Soc., 293, 1984.

[6] Jean-lin Journé, Calderón-Zygmund operators, Pesudo-differential operators and the Cauchy integral of Calderón, Lecture Notes in Math., 994 (1983), Spring-Verlag, Berlin.

[7] S. Janson, Mean oscillation and commutators of singular integral operators, Ark. Math., 16 (1978), 263-270.

[8] W. M. Li and C. R. Xu, Lipschitz Function spaces on spaces of Homogeneous type, Acta Anal. Func. Appl., 5 (2003), 369-373.

[9] S. Z. Lu and D. C. Yang, The decomposition of Herz space on local fields and its applications, J. of Math. Anal. and Appl., 196 (1995), 296-313.

[10] M. Paluszyński, Characterization of the Besov spaces via the commutator operator of Coifman, Rochbery and Weiss, Indiana Univ. Math. J., 44 (1995), 1-17.

[11] R. A. Macias and S. Carlos, Lipschitz function on spaces of Homogeneous type, Advance in Math., 33 (1979), 257-276.

[12] A. Uchiyama, On the compactness of operators of Hankel type, Tôhoku Math. J., 30 (1978), 163-171.

[13] J. S. Xu and D. C. Yang, Herz-type Triebel-Lizorkin space (I), Acta. Math. Scinia., to appear.

[14] Y. Yabuta, Generalization of Calderón-Zygmund operators, Studia Math., 82 (1985), 17-31. 
222 Boundedness of commutators on locally compact Vilenkin groups

Department of Mathematics

Hunan University

Changsha, Hunan

P. R. China

and

Department of Mathematics

Hunan University of Arts and Science

Changde, Hunan

P. R. China

(E-mail : tangcq2000@yahoo.com.cn)

Department of Mathematics

Hunan University

Changsha, Hunan

P. R. China

(E-mail : liqingguoli@yahoo.com.cn)

Department of Mathematics

Hunan University

Changsha, Hunan

P. R. China

(E-mail : blma@hnu.net.cn)

(Received: August 2004) 


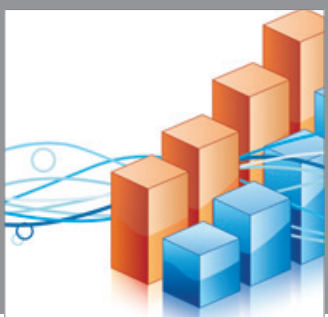

Advances in

Operations Research

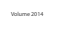

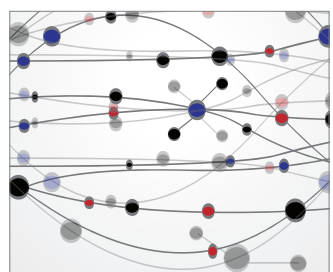

\section{The Scientific} World Journal
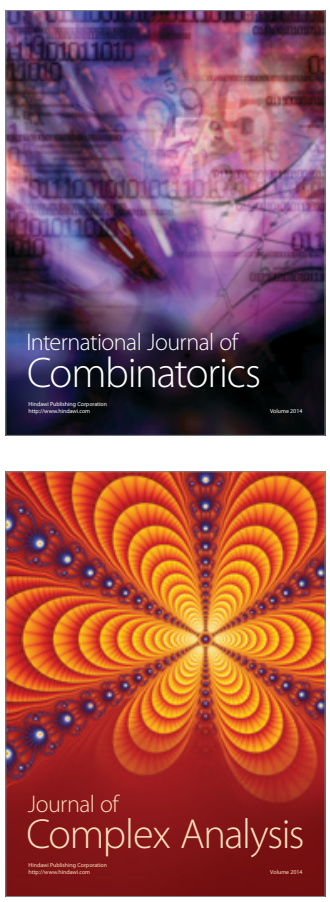

International Journal of

Mathematics and

Mathematical

Sciences
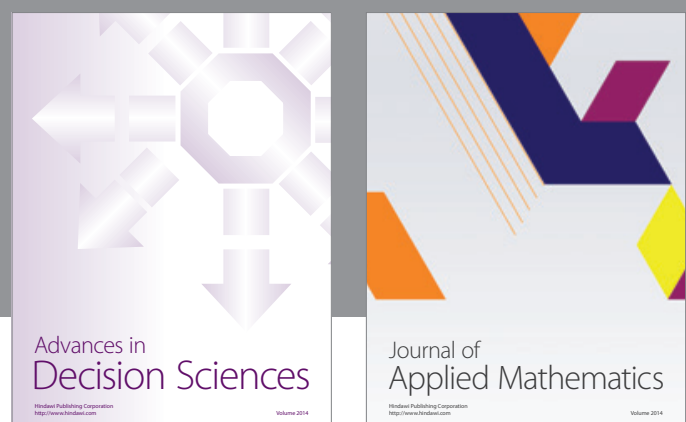

Journal of

Applied Mathematics
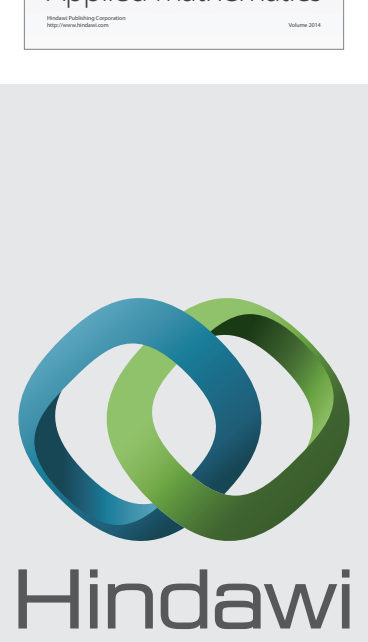

Submit your manuscripts at http://www.hindawi.com
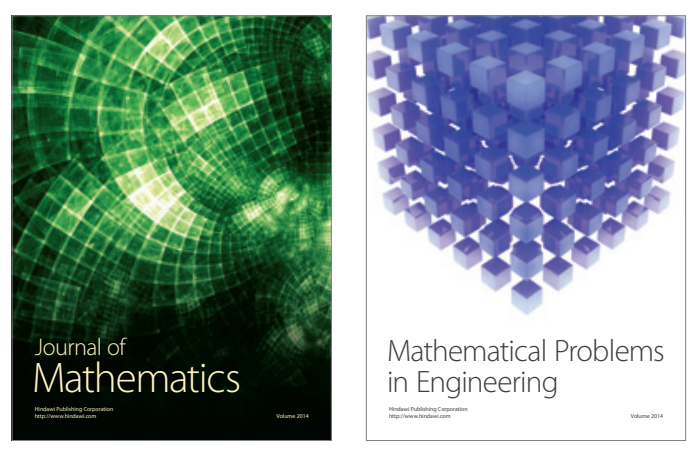

Mathematical Problems in Engineering
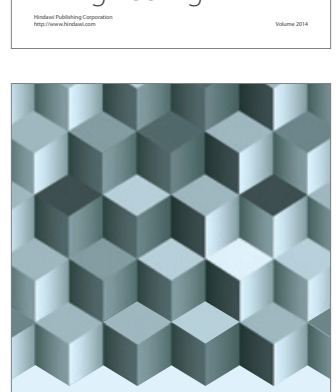

Journal of

Function Spaces
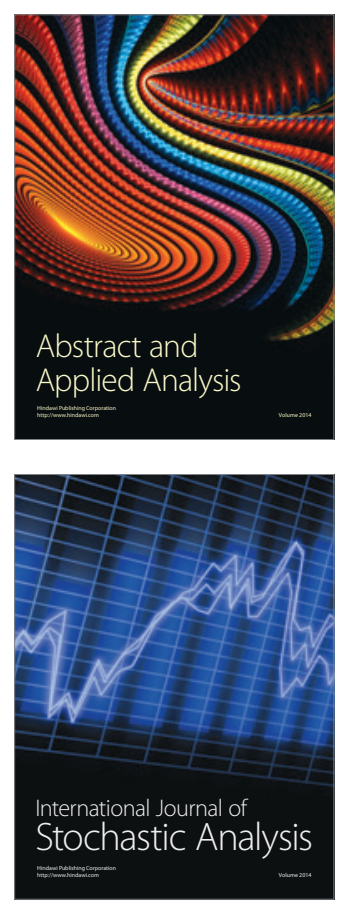

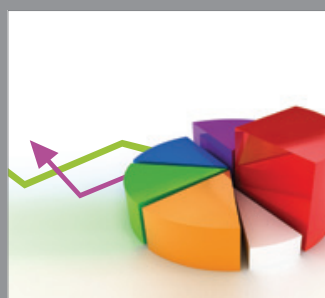

ournal of

Probability and Statistics

Promensencen
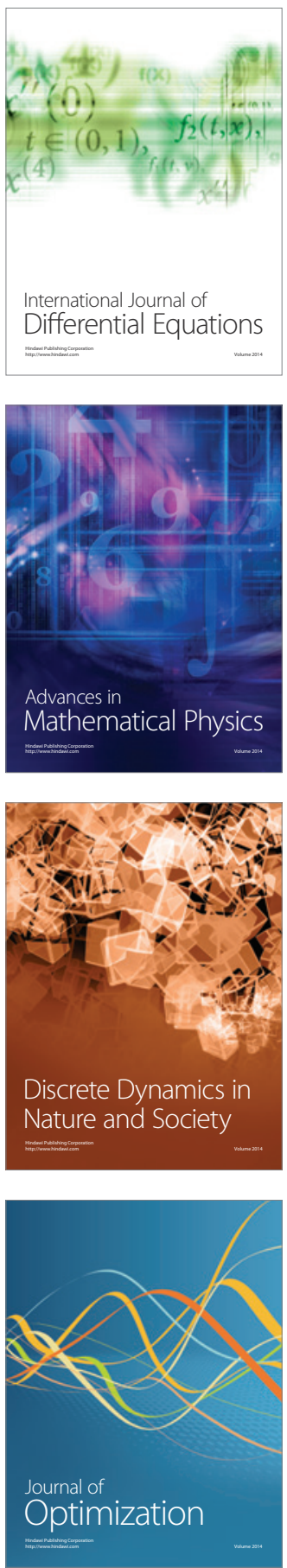